Review

\title{
Food Rescue Intervention Evaluations: A Systematic Review
}

\author{
Amelie A. Hecht $1,2, *(1)$ and Roni A. Neff ${ }^{1,2,3}$ \\ 1 Department of Health Policy and Management, Johns Hopkins Bloomberg School of Public Health, \\ Baltimore, MD 21205, USA; rneff1@jhu.edu \\ 2 Center for a Livable Future, Johns Hopkins Bloomberg School of Public Health, Baltimore, MD 21202, USA \\ 3 Department of Environmental Health and Engineering, Johns Hopkins Bloomberg School of Public Health, \\ Baltimore, MD 21205, USA \\ * Correspondence: ahecht3@jhu.edu
}

Received: 24 October 2019; Accepted: 17 November 2019; Published: 27 November 2019

check for updates

\begin{abstract}
Food rescue, the practice of gathering food that could otherwise be wasted and redirecting it for human consumption, represents a critical opportunity to improve food security and reduce waste. As global interest in reducing hunger and food waste grows, better insight is needed to assess and compare the effectiveness of different models of food rescue. We conducted a systematic review of peer-reviewed studies evaluating food rescue interventions with the aim of synthesizing findings and comparing methodologies. We searched PubMed, Academic Search Ultimate, and Science Citation Index for studies published worldwide, in English, through June 2019. Studies were included that: a) evaluated an existing or proposed food rescue intervention and, b) quantitatively or qualitatively measured the intervention impact. All nineteen included studies were observational and the intervention types ranged widely. The most commonly reported metric was the weight of food recovered. Few studies reported client outcome measures. The included studies suggested promising effects of food rescue interventions, including positive return on investment, decreased environmental burden, large quantities of food rescued and clients served, and high stakeholder satisfaction. Comparison across studies was challenging, however, due to inconsistent metrics and insufficiently detailed methodology. This review documents a need for additional evaluation of food rescue interventions and recommends a standardized methodology. Additional dialogue among key stakeholders is warranted to develop consistent, meaningful metrics to assess food rescue.
\end{abstract}

Keywords: food recovery; food rescue; food redistribution; wasted food; food security; methodology

\section{Introduction}

Food rescue, sometimes referred to as food recovery or redistribution, is the practice of gathering rescuable food and redirecting it for human consumption. While there is no universal criterion for rescuability, the term refers to edible food which is currently safe to eat and of good quality; rescuable food may contain inedible parts [1]. Within the U.S. Environmental Protection Agency's Food Recovery Hierarchy, which prioritizes actions to prevent and divert wasted food, feeding hungry people through food rescue ranks just below source reduction [2].

Food rescue represents a critical opportunity in a food system with high levels of both waste and food insecurity. An estimated one third of the global food supply is lost or wasted, amounting to approximately 1.3 billion tons per year [3], while at the same time, 2 billion people, $26 \%$ of the world's population, experience moderate or severe food insecurity [4]. No identified reports estimate the amount of food rescued globally and only one identified report estimates the amount of food rescued within a specific country. That report, a 2016 analysis published by ReFED, a multi-stakeholder 
organization addressing wasted food, estimated that 1.7 million tons of food are rescued annually in the U.S., $64 \%$ of which comes from retail, $27 \%$ from farms, and $9 \%$ from restaurants and foodservice [5].

While a substantial portion of food that is wasted is inappropriate for rescue due to factors including spoilage, logistics, and a lack of cost effectiveness, ReFED estimated that in the U.S., it would be possible to recover triple the amount currently recovered—an additional 5.8 million tons of edible food [5]. Walia and Sanders estimated that rescuing enough food to reduce edible waste in the U.S. by $15 \%$ would provide full sustenance for 18.5 million people, or alternately, $35 \%$ of food needs for every American living with food insecurity [6]. Food rescue is not the primary solution for food insecurity or surplus. Root cause solutions are more effective and lasting and provide co-benefits in well-being and resource use. Nonetheless, even in a best-case prevention scenario, an essential role remains for programs connecting available food to those who need it.

Food rescue systems are complex. Figure 1 presents an overview of the range of food rescue pathways and destinations including food sources, reasons, rescuers, and distributors.

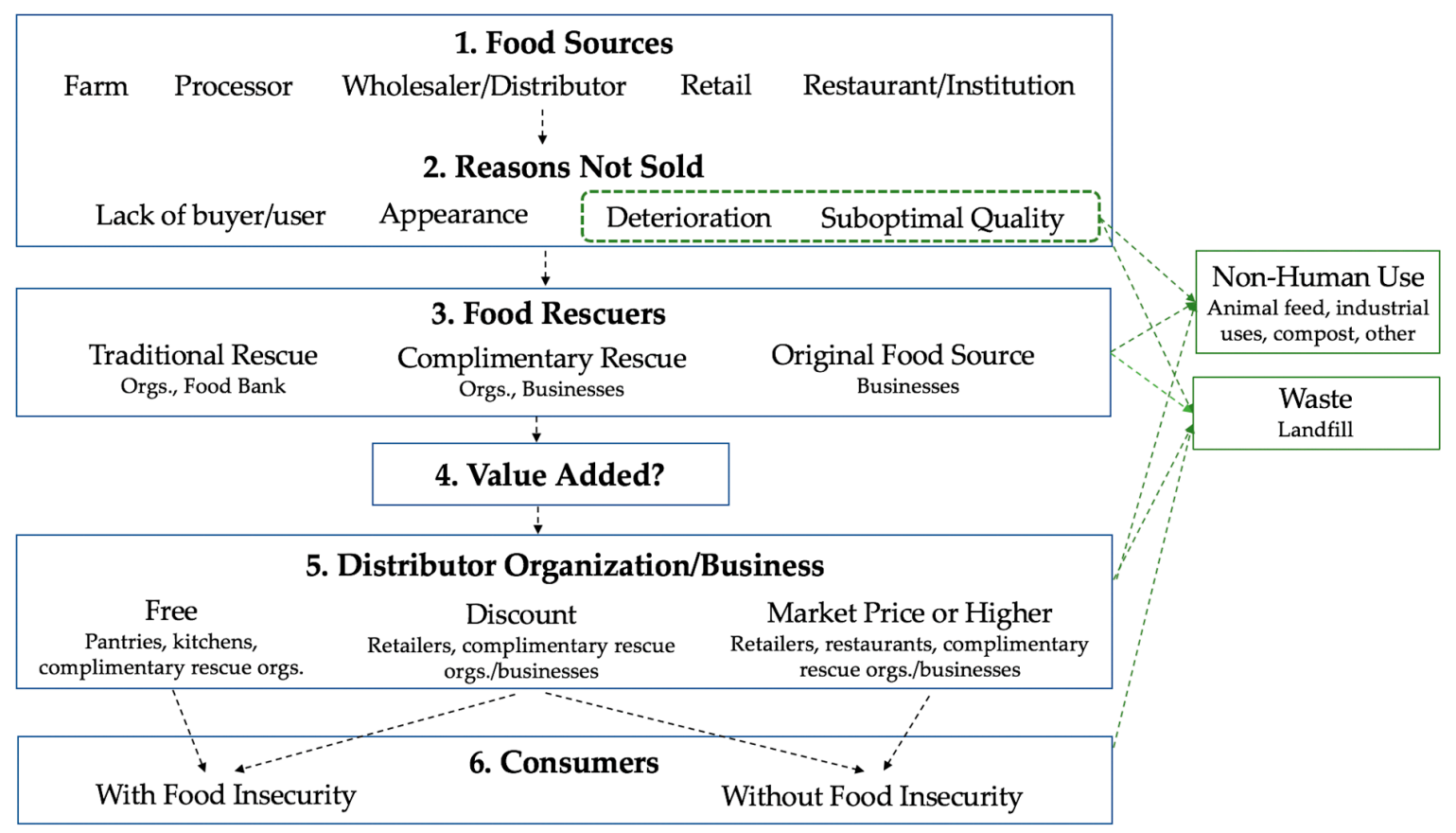

Figure 1. Rescuable food pathways and destinations.

1. Food sources: Rescuable food may originate anywhere in the food supply chain, including farms and producers, processors, wholesalers/distributors, retailers, or restaurants and institutions. Smaller quantities of food can be rescued from homes, such as through canned food drives.

2. Reasons: Four categories cover the range of proximal causes for food not being sold: lack of buyer/user, appearance, deterioration, and suboptimal quality [7]. The food in the latter two categories may not be fit for human consumption but can be recovered for non-human uses.

3. Rescuers: Three main organization types perform food rescue. What we call "traditional" rescue organizations, such as food banks, gather the largest amount. These have historically focused mainly on shelf-stable foods, although this is changing. Diverse "complementary" rescue organizations address gaps in the system (perceived and actual). ReFED's Food Waste Innovator Database [8] identifies 100 food rescue projects addressing donation coordination, transportation, and processing. These include services and apps to manage donation and delivery logistics; programs creating value-added food products such as soup for donation; programs to collect food from sources not often included in food rescue such as farms, farmers markets or public events; employment programs; and programs gathering "ugly" and distressed products. The original 
food source itself can also serve as rescuer, by connecting directly to distributors, or implementing alternative sale strategies such as discounting older foods.

4. Value-added: In some cases, value-added preparation is performed before distribution, such as processing food or scraps into soups or jams.

5. Distributors: Both traditional (e.g., food pantry, kitchen) and complementary (e.g., business selling boxes of rescued food) interventions distribute rescued food, including offering it for free, selling at discount, or selling at market price/markup.

6. Consumers: Much rescued food goes to consumers with food insecurity, but as concern grows regarding waste, consumers without food insecurity also seek this food supply.

The route taken by rescuable food depends on factors including the food type and value, and local context. While the charitable food sector plays a major role in the U.S., countries with strong social welfare systems have less need for food pantries and food banks, making retailing strategies such as discount sales more common. ("Traditional" charitable food in those countries may be more commonly used by groups falling through the system's cracks, such as refugees.)

We also note that anywhere along this rescue chain, food that is of lower quality or has spoiled or passed its date label is diverted to non-human uses or waste. Well-meaning efforts to prevent waste may inadvertently result in donation of near-spoiled food, thus shifting the burden and cost of addressing waste onto the food rescue system and consumers with lower incomes rather than the food source business.

A small descriptive literature has examined barriers and challenges in advancing food rescue, primarily in high-income countries, and largely based on interviews, surveys, and case studies. Key challenges identified include: administrative and logistical barriers; limited funds, space and other resources; staffing limitations; need for improved networks and relationships; need for information about how to donate food; and need for improved donation quality [5,6,9-15].

Little is known about the impacts of food rescue interventions, which models of food rescue are most successful, how to improve intervention effectiveness, and what may be needed to transfer insights from one intervention to another or to scale interventions. The lack of evaluation and dissemination also contributes to a situation in which seemingly novel programs frequently replicate existing approaches, expending considerable effort and expense relearning lessons, redeveloping similar systems, and at times competing rather than collaborating.

This systematic review aims to synthesize insights from existing peer-reviewed literature evaluating food rescue programs and to make recommendations regarding the measurement and consistent methodology, to provide a starting place for advancing future evaluation research. This review complements existing systematic reviews of literature on food waste prevention [16] and life cycle assessment studies [17].

\section{Materials and Methods}

Searches were conducted for empirical, peer-reviewed articles on food rescue interventions, published worldwide, in English, through June 2019. The following keyword combinations were used to search electronic databases (PubMed, Academic Search Ultimate and Science Citation Index): ("food gleaning" NOT bat) OR ("food redistribution") OR ("food recovery") OR ("food rescue") OR ("food donat") OR ("donated food"). The reference lists of relevant articles and reviews were also examined. Preferred Reporting Items for Systematic Reviews and Meta-Analysis (PRISMA) guidelines were used to track and report articles identified through the literature search [18].

Inclusion criteria were: a) the evaluation of an existing or proposed food rescue intervention, $b$ ) quantitatively or qualitatively measured impact of a food rescue intervention. Articles that did not meet inclusion criteria were excluded. Articles that focused only on mathematical simulation of delivery models (i.e., simulated how to best design rescue pick-up and drop-off systems for maximum efficiency) or only process evaluation (e.g., description of food rescue operations, barriers to implementation) but 
did not quantitatively or qualitatively measure impact of a specific intervention were excluded from the literature synthesis. Many of the excluded articles that focused on process evaluation or provided an overview of the food rescue landscape were incorporated in the background and discussion sections of this article.

In addition to articles meeting study inclusion criteria, the search strategy identified nine articles providing useful context on barriers and challenges to food rescue and donation. To create a prioritization of concerns that might be addressed through interventions, barriers and challenges mentioned in these articles were enumerated and then sorted and ranked based on frequency (Table 1). Some articles mentioned multiple distinct barriers and challenges that fit within a single category, such as two different administrative and logistical barriers; these were counted separately.

Table 1. Top barriers to food rescue identified in prior research.

\begin{tabular}{|c|c|c|c|}
\hline Barrier & Example & Reference (Author/s, Date) & Frequency \\
\hline $\begin{array}{l}\text { Need for improved } \\
\text { relationships and } \\
\text { communication between } \\
\text { donor and recipient } \\
\text { organizations }\end{array}$ & $\begin{array}{l}\text { - Relationships require considerable investment and } \\
\text { communication to work effectively and ensure that } \\
\text { recipient needs are communicated to donors. } \\
\text { Tension between creating systematized procedures } \\
\text { for efficiency versus the benefit of individualized } \\
\text { relationships; such systems may exclude smaller } \\
\text { anti-hunger organizations. } \\
\text { Competing priorities, disorganization and other } \\
\text { factors can make it challenging to engage key players. }\end{array}$ & $\begin{array}{l}\text { Benson et al., 2018; } \\
\text { Hermsdorf et al., 2017; Otten } \\
\text { et al., } 2018\end{array}$ & 6 \\
\hline $\begin{array}{l}\text { Need for information } \\
\text { about how to donate } \\
\text { food and maintain food } \\
\text { safety }\end{array}$ & $\begin{array}{l}\text { There is need for educational materials about how to } \\
\text { donate, how to preserve food safety, and } \\
\text { available incentives. }\end{array}$ & $\begin{array}{l}\text { Benson et al. 2018; Bierma et } \\
\text { al., 2019; Hermsdorf et al., } \\
\text { 2017; Otten et al., } 2018\end{array}$ & 4 \\
\hline $\begin{array}{l}\text { Need for improved } \\
\text { donation food quality }\end{array}$ & $\begin{array}{l}\text { - Some items donated cannot be sold. } \\
\text { - Some items of low nutritional quality are donated. }\end{array}$ & $\begin{array}{l}\text { Tarasuk et al., 2005; } \\
\text { Wingrove et al., } 2017\end{array}$ & 2 \\
\hline
\end{tabular}




\section{Results}

The search yielded 345 results. (Figure 2) An additional four articles were identified outside the formal search process by searching the reference lists of included articles. After removing duplicates, 272 abstracts were screened, and 80 articles were subject to full-text review. Sixty-one studies that did not meet eligibility criteria were excluded. Nineteen studies were included in this review. Table 2 summarizes the locations of included studies and provides brief descriptions of the interventions, and Table 3 describes the study design and food rescue metrics and presents findings on program impacts.
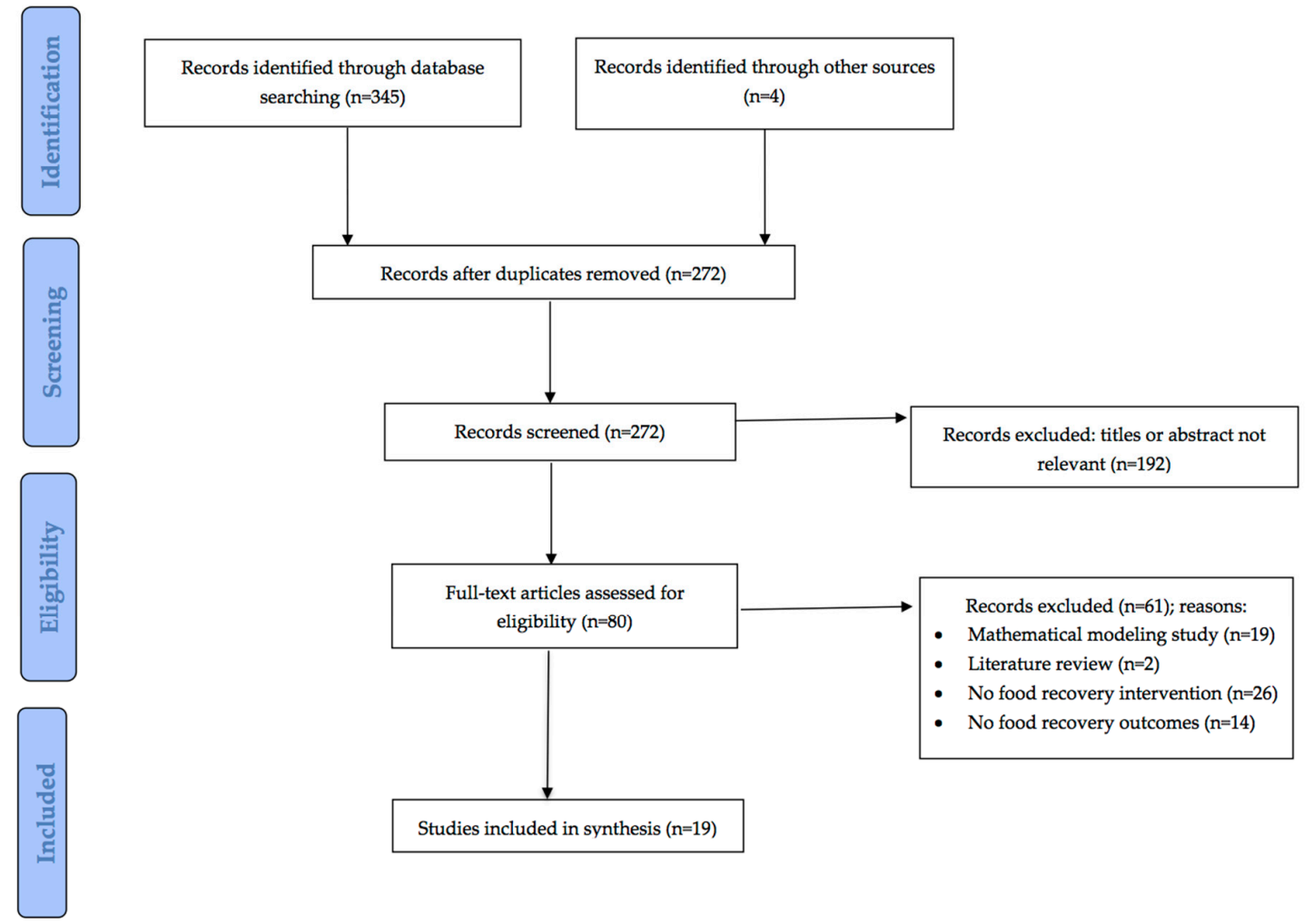

Figure 2. Preferred Reporting Items for Systematic Reviews and Meta-Analysis (PRISMA) Diagram tracks which articles were identified and included through the literature search.

All included studies occurred in high-income countries, including six from Italy, five from the U.S., two from Australia, two from the U.K., and one each from Belgium, Finland, Israel, and New Zealand.

Intervention types ranged widely, including interventions such as retail food redistribution, gleaning from fields and farmers markets, and leftover school lunch programs. Following the typology of food rescue initiatives established by ReFED [8], eight studies focused on donation transportation interventions (i.e., organizations actively involved in the collection and distribution of donated food), six studies focused on donated food processing interventions (i.e., organizations that collect, store, and process donated food for distribution, such as community kitchens and value-added processing), two studies focused on organizations involved in donated food coordination (i.e., organizations that enable aspects of food donation, including matching food sources with rescuers and distributors), and three studies included organizations involved in both donation transportation and donated food processing interventions. 
Table 2. Location and intervention description of included studies $(n=19)$.

\begin{tabular}{|c|c|c|}
\hline Reference (Author/s, Date) & Geography & Intervention and Sample Description \\
\hline Alexander et al., 2008 & Southampton, UK & Food rescued primarily from two retailers, distributed to two centers that serve homeless clients. \\
\hline Bonaccorsi et al., 2016 & Florence, Italy & Donated food at a charitable organization frozen to lengthen its shelf life. \\
\hline Cicatiello et al., 2016 & Viterbo, Italy & $\begin{array}{c}\text { Food rescued from one supermarket, transported to soup kitchens by volunteers, and prepared for } \\
\text { clients. }\end{array}$ \\
\hline Cicatiello et al. 2017 & Italy & Food rescued from one supermarket, transported to charities. \\
\hline De Boeck et al., 2017 & Flanders, Belgium & Food donated to four food banks or local charity organizations. \\
\hline Deavin et al., 2018 & New South Wales, Australia & $\begin{array}{l}\text { Free primary school-based breakfast program using donated food, served once per week for two } \\
\text { terms in one school. }\end{array}$ \\
\hline Facchini et al., 2018 & London, UK & Non-exhaustive list of 12 food redistribution organizations in the city. \\
\hline Garcia-Silva et al., 2017 & Orange County, California, US & $\begin{array}{l}\text { County-wide coalition to mitigate hunger through education about food donations, identification } \\
\text { of food-insecure individuals, and connecting those individuals to sources of food. }\end{array}$ \\
\hline Hoisington et al., 2001 & Pierce County, Washington, US & $\begin{array}{l}\text { Pierce County Expanded Food and Nutrition Education Program Gleaning Project engaging } 50 \\
\text { gleaners working in fields and orchards. }\end{array}$ \\
\hline Laakso, 2017 & Jyväskylä, Finland & $\begin{array}{l}\text { After the school lunch service, retired and unemployed people invited to consume a "leftover } \\
\text { lunch". }\end{array}$ \\
\hline Milicevic et al., 2016 & Italy & Food rescued from catering event for distribution to food bank. \\
\hline Mirosa et al., 2016 & New Zealand & $\begin{array}{l}\text { Food redistribution organization that distributes surplus food from local business to charitable } \\
\text { agencies (FoodShare). }\end{array}$ \\
\hline Moggi et al., 2018 & Italy & Farmers market food rescue program using a network-level activity system. \\
\hline Mousa et al., 2017 & Eight Southwestern states, US & One hundred organizations involved in food rescue nutrition. \\
\hline Philip et al., 2017 & Israel & $\begin{array}{l}\text { Business-to-business food bank that redistributes perishable food obtained from an agricultural } \\
\text { gleaning project, self-growing farm project, and meal rescue projects. }\end{array}$ \\
\hline Reynolds et al., 2015 & Australia & $\begin{array}{l}\text { Four food rescue organizations analyzed in the context of the entire Australian waste treatment } \\
\text { industry. }\end{array}$ \\
\hline Sewald et al., 2018 & Boulder, Colorado, US & $\begin{array}{l}\text { Boulder Food Rescue uses web-application "robot" to manage volunteers and food rescue } \\
\text { deliveries. }\end{array}$ \\
\hline Sisson, 2016 & Grand Rapids, Michigan, US & $\begin{array}{l}\text { Low-income volunteers redistribute fresh produce from farmers' markets to individuals and food } \\
\text { pantries. }\end{array}$ \\
\hline Vittuari et al., 2017 & Emilia Romagna, Italy & $\begin{array}{l}\text { Sixty-one food distribution initiatives ranging from food banks and pantries to shelters and } \\
\text { religious or civic organizations. }\end{array}$ \\
\hline
\end{tabular}


Table 3. Study design, food rescue metrics, impact measures for included studies $(n=19)$.

\begin{tabular}{|c|c|c|c|c|c|}
\hline Reference & Study Period & Methods & Sample & Key Metrics* & Impact \\
\hline Alexander et al., 2008 & Two days & $\begin{array}{l}\text { Measurement triangulated } \\
\text { with interviews with } \\
\text { officials from the charity } \\
\text { headquarters, recipient } \\
\text { charities and donating } \\
\text { retailers }\end{array}$ & $\begin{array}{l}\text { Two retailers; unreported } \\
\text { number of interviews }\end{array}$ & $\begin{array}{ll}\text { Across } 2 \text { days: } \\
: \quad \text { Weight of food donated } \\
-\quad \text { Weight of food accepted } \\
\dot{-} \quad \text { Weight of food delivered } \\
\quad \text { Weight of food served } \\
\quad \text { Weight of food consumed }\end{array}$ & 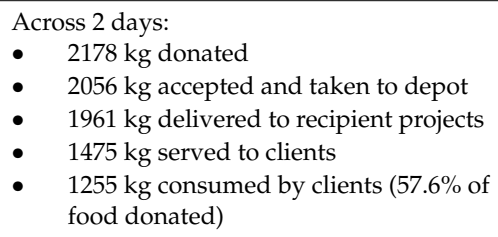 \\
\hline Bonaccorsi et al., 2016 & $\begin{array}{l}\text { Sampled products had } \\
\text { been frozen for } 45 \text { days, } \\
\text { thawed for } 48 \text { hours and } \\
\text { cooked within } 24 \text { hours }\end{array}$ & $\begin{array}{l}\text { Microbiological sample } \\
\text { analysis }\end{array}$ & 90 food samples & $\begin{array}{l}\text { Across } 90 \text { food samples: } \\
\text { - Total aerobic microbial count } \\
\text { - Presence of Escherichia coli, Salmonella } \\
\text { spp, Staphylococcus aureus, } \\
\text { Campylobacter spp, Sulphite } \\
\text { reducing clostridia }\end{array}$ & $\begin{array}{l}\text { Across } 90 \text { food samples: } \\
\text { - Almost all cooked product risk profiles } \\
\text { represented no hazard } \\
\text { No or non-hazardous levels detected of } \\
\text { Escherichia coli, Salmonella spp, } \\
\text { Campylobacter spp, Staphylococcus } \\
\text { aureus, Sulphite reducing clostridia }\end{array}$ \\
\hline Cicatiello et al., 2016 & One year & Measurement & One supermarket & $\begin{array}{l}\text { Over one year: } \\
\text { - Number of deliveries (total and } \\
\text { avg./month) } \\
\text { - Weight of food rescued (total and } \\
\text { avg./delivery) } \\
\text { - Categories of food rescued (e.g., bread) } \\
\text { - Economic value of rescued food (based } \\
\text { on retail price) } \\
\text { Environmental value of rescued food } \\
\text { (water and ecological footprint of } \\
\text { rescued food) } \\
\text { - Possible number of servings prepared } \\
\text { with rescued food } \\
\text { Cost-revenue estimate }\end{array}$ & $\begin{array}{l}\text { Over one year: } \\
\text { - } \quad 300 \text { deliveries (avg. } 25 / \text { month) } \\
23.5 \text { tons of food rescued (avg. } \\
80 \mathrm{~kg} / \text { delivery) } \\
\text { - } \quad \text { Bread accounted for more than } 70 \% \text { of } \\
\text { the weight of total recoveries } \\
\quad € 46,000 \text { of food rescued } \\
\quad 30,294 \text { cubic meters of water associated } \\
\text { with rescued meat (other example } \\
\text { given for bread) } \\
\text { - } \quad \text { Rquivalent of } 3624 \text { meals rescued } \\
\quad \text { Return on investment was } 4 \times \text { larger } \\
\quad \text { than the investment needed to start and } \\
\text { run the project }\end{array}$ \\
\hline Cicatiello et al., 2017 & One year & Measurement & One supermarket & $\begin{array}{l}\text { Over one year: } \\
\text { - Weight of food rescued (overall and } \\
\text { - } \quad \text { Ecrcent by food category) } \\
\text { on retail price) }\end{array}$ & $\begin{array}{l}\text { Over one year: } \\
\text { - } 24.6 \text { tons of food rescued }(34.5 \% \text { of food } \\
\quad \text { waste generated was rescued) } \\
\quad € 37,644 \text { of food rescued ( } 22.2 \% \text { of food } \\
\text { wasted generated) }\end{array}$ \\
\hline
\end{tabular}


Table 3. Cont

\begin{tabular}{|c|c|c|c|c|c|}
\hline Reference & Study Period & Methods & Sample & Key Metrics* & Impact \\
\hline De Boeck et al., 2017 & $\begin{array}{l}\text { Seven weeks; at each visit, } \\
\text { two to ten food samples } \\
\text { were taken from each } \\
\text { recipient organization }\end{array}$ & $\begin{array}{l}\text { Microbiological sample } \\
\text { analysis }\end{array}$ & 72 food samples & $\begin{array}{l}\text { Across } 72 \text { samples: } \\
\text { - } \quad \text { Quality indicators } \\
\text { - } \quad \text { Hygiene indicators } \\
\text { - }\end{array}$ & $\begin{array}{l}\text { Across } 72 \text { samples: } \\
\text { - } \quad 22 \text { samples showed marginal } \\
\text { microbiological quality } \\
\text { - } \quad \text { Listeria monocytogenes detected in } \\
\text { three samples } \\
\text { - One sample showed high levels of } \mathrm{L} \text {. } \\
\text { monocytogenes and Enterobacteriaceae }\end{array}$ \\
\hline Deavin et al., 2018 & $\begin{array}{l}\text { Two school terms; food } \\
\text { diaries completed over } 5 \\
\text { days during intervention } \\
\text { period }\end{array}$ & Focus groups & $\begin{array}{l}21 \text { students, six teachers, } \\
\text { two parents }\end{array}$ & $\begin{array}{l}\text { Over two school terms: } \\
-\quad \text { Weight of food rescued } \\
\text { - } \quad \text { Direct cost of the program } \\
\text { - } \quad \text { Pelunteer hours } \\
\quad \text { parentions of teachers, students and } \\
\text { and impact } \\
\quad \text { Equivalent meals rescued }\end{array}$ & $\begin{array}{l}\text { Over two school terms: } \\
\text { - } \quad 4.4 \text { tons food rescued } \\
\text { - } \quad \text { conso,340 in project cost, including } \\
\text { - } \quad 4708 \text { volunteer host salary and van } \\
\text { - } \quad \text { Positive perceived impacts on student } \\
\quad \text { focus and alertness, confidence, energy, } \\
\text { school attendance, knowledge and skill } \\
\text { development, community engagement, } \\
\text { food security } \\
\text { - } \quad 44,000 \text { meals rescued }\end{array}$ \\
\hline Facchini et al., 2018 & Cross-sectional in 2014 & $\begin{array}{l}\text { Interviews with food } \\
\text { redistribution organization } \\
\text { managers }\end{array}$ & $\begin{array}{l}\text { Unreported number of } \\
\text { interviews; } 12 \text { example } \\
\text { food redistribution sites }\end{array}$ & $\begin{array}{l}\text { - Weight of food rescued (using various } \\
\text { denominators, e.g., annually, per event) } \\
\text { - Sources of rescued food } \\
\text { - Org. activities (e.g., gleaning, } \\
\text { serving dinner) }\end{array}$ & $\begin{array}{l}\text { - Variation in amount of food rescued by } \\
\text { each org. } \\
\text { - Variation in sources of food (e.g., } \\
\text { supermarkets, producers, } \\
\text { wholesalers, events) } \\
\text { - } \quad \text { Variation in org. activities (e.g., turn } \\
\text { surplus fruit into fruit jerky, serve } \\
\text { vegan dinners) }\end{array}$ \\
\hline Garcia-Silva et al., 2017 & 22 months & Measurement & One county coalition & $\begin{array}{l}\text { Over } 22 \text { months: } \\
\text { - } \quad \text { Weight of food rescued } \\
\text { - } \quad \text { Meals rescued } \\
\text { - } \quad \text { Donor agencies recruited } \\
\quad \text { Recipient agencies (pantries) recruited }\end{array}$ & $\begin{array}{l}\text { Over } 22 \text { months: } \\
\text { - } \quad 280 \text { tons food rescued } \\
\text { - } \quad \sim 466,637 \text { meals rescued } \\
\text { - } \quad 50 \text { donors recruited } \\
\quad 25 \text { pantries recruited }\end{array}$ \\
\hline
\end{tabular}


Table 3. Cont.

\begin{tabular}{|c|c|c|c|c|c|}
\hline Reference & Study Period & Methods & Sample & Key Metrics* & Impact \\
\hline Hoisington et al., 2001 & $\begin{array}{l}\text { One farm season } \\
\text { (June-November) }\end{array}$ & $\begin{array}{l}\text { Measurement over one farm } \\
\text { season, survey tracking } \\
\text { 4-week use of produce by } \\
\text { subsample of gleaners }\end{array}$ & $\begin{array}{l}50 \text { gleaners observed; } \\
\text { subsample of } 29 \text { surveyed }\end{array}$ & $\begin{array}{l}\text { - } \quad \text { Weight of food gleaned } \\
\text { - } \quad \text { taken hom of food gleaned donated vs. } \\
\text { - Use of produce taken home by gleaners } \\
\text { - } \quad \text { Perceived benefits of gleaning } \\
\text { among participants }\end{array}$ & $\begin{array}{l}\text { Across } 50 \text { gleaners: } \\
\text { - } \quad 110,000 \text { pounds gleaned } \\
\text { - } 85,000 \text { pounds }(77 \%) \text { donated to local } \\
\text { emergency food programs and } 25,000 \\
\text { pounds }(23 \%) \text { taken home by gleaners } \\
\text { - } \quad \text { Across } 29 \text { gleaners: } \\
\text { Of the produce taken home, } 9 \% \text { was } \\
\text { used fresh, } 48 \% \text { was pre-served for later } \\
\text { user, and } 43 \% \text { was shared with others } \\
\text { Benefits cited included improved diet, } \\
\text { shared or received knowledge of } \\
\text { gardening, preservation and nutrition, } \\
\text { helping the community, stretching } \\
\text { food budget }\end{array}$ \\
\hline Laakso, 2017 & Cross-sectional in 2015 & $\begin{array}{l}\text { Interviews with diners at } \\
\text { "leftover lunch" service } \\
\text { across three schools }\end{array}$ & 3 schools; 24 diners & $\begin{array}{l}\text { Diner perspectives on how the leftover } \\
\text { lunch has impacted their daily routine, } \\
\text { and how important the meal is to them }\end{array}$ & $\begin{array}{l}\text { - Meal is a daily habit and is main meal } \\
\text { of the day } \\
\text { - Lunch is affordable and nutritious } \\
\text { - Lunch is important socially }\end{array}$ \\
\hline Milicevic et al., 2016 & Four days & $\begin{array}{l}\text { Microbiological sample } \\
\text { analysis }\end{array}$ & $\begin{array}{l}44 \text { total samples (11 food } \\
\text { items each sampled daily) }\end{array}$ & $\begin{array}{l}\text { Hygienic status of food rescued at four time } \\
\text { points and under several different } \\
\text { preservations terms }\end{array}$ & $\begin{array}{l}\text { Across } 11 \text { foods sampled } 4 \mathrm{x} \text { each: } \\
\text { - } 18.8 \% \text { of total food samples had } \\
\text { presence of Listeria monocytogenes } \\
\text { and Salmonella spp. } \\
\text { - One sample contained } \\
\text { Enterobacteriaceae and Bacillus cereus }\end{array}$ \\
\hline Mirosa et al., 2016 & Cross-sectional in 2015 & $\begin{array}{l}\text { Interviews with FoodShare } \\
\text { staff and stakeholder } \\
\text { organizations (food donors, } \\
\text { financial donors, recipient } \\
\text { agencies, volunteers); } \\
\text { survey of volunteers }\end{array}$ & $\begin{array}{l}\text { Interviews: } 13 \text { (two } \\
\text { FoodShare staff and } 11 \\
\text { stakeholder } \\
\text { organizations); survey: } 40 \\
\text { volunteers }\end{array}$ & $\begin{array}{l}\text { Impact of participation in FoodShare on: } \\
\text { - } \quad \text { Food donors } \\
\text { - Financial donors } \\
\text { - } \quad \text { Recipient agencies } \\
\quad \text { Volunteers }\end{array}$ & $\begin{array}{l}\text { Impact of participation in FoodShare on: } \\
\text { - Food donors: improved donor image } \\
\text { and community relationships } \\
\text { - Financial donors: promotion } \\
\text { opportunity, helping others } \\
\text { - Recipient agencies: increased food } \\
\text { volume, nutritional quality and } \\
\quad \text { program reach } \\
\text { Volunteers: meeting new people, new } \\
\text { skills, sense of accomplishment }\end{array}$ \\
\hline
\end{tabular}


Table 3. Cont

\begin{tabular}{|c|c|c|c|c|c|}
\hline Reference & Study Period & Methods & Sample & Key Metrics* & Impact \\
\hline Moggi et al., 2018 & 2009-2018 & $\begin{array}{l}\text { Measurement and } \\
\text { document review }\end{array}$ & One farmers market & $\begin{array}{l}\text { - Weight of food rescued } \\
\text { - } \quad \text { Sconomic value of food rescued } \\
\text { - } \quad \text { Sampost costs } \\
\text { - } \quad \text { Famings of } \mathrm{CO} 2 \text { emissions } \\
\end{array}$ & $\begin{array}{l}\text { - In } 2013,790 \text { tons of fruit and } \\
\text { vegetables rescued } \\
\text { - In 2013, economic value of } 900,000 \\
\text { euros food rescued } \\
\text { - Annual growth of approx. } 25 \% \\
\text { food donated } \\
\text { - In 2017, approx. } 140,000 \text { euros saved in } \\
\text { collection costs, } 30,000 \text { in disposal costs, } \\
80,000 \text { in compost cost } \\
\text { - In } 2017 \text {, approx. } 3000 \text { tons of } \mathrm{CO} 2 \text { saved } \\
\text { - In 2015, 3000 families provided } \\
\text { food daily }\end{array}$ \\
\hline Mousa et al., 2017 & Cross-sectional in 2015 & $\begin{array}{l}\text { Survey of organization } \\
\text { directors }\end{array}$ & $\begin{array}{l}100 \text { food rescue } \\
\text { organizations }\end{array}$ & $\begin{array}{ll}\text { - } & \text { Weight of rescued food served } \\
\text { (per month) } \\
\text { - } \quad \text { Clients served (per month) } \\
\text { - } \quad \text { Number of employees and volunteers } \\
\text { - }\end{array}$ & $\begin{array}{l}\text { Across } 100 \text { organizations: } \\
\text { - } \quad \text { On million in food benefits distributed } \\
\text { - } \quad \text { Served } 2 \text { million kg of food per month } \\
\text { - } \quad \text { Served more than } 40,000 \text { clients/month } \\
\text { - Employed eight workers and } 3081 \\
\quad \text { volunteers (serving an average of } 16 \\
\quad \text { hours each) }\end{array}$ \\
\hline Philip et al., 2017 & Three years & Measurement & One food bank & $\begin{array}{l}\text { Over one year: } \\
\text { - Weight of agricultural surplus rescued } \\
\text { - } \quad \text { Peight of food redistributed } \\
\text { - } \quad \text { fruits/vegetion of food that is healthy, } \\
\text { - } \quad \text { Numbersity of food delivered } \\
\text { - } \quad \text { partner or nonponizations } \\
\text { - } \quad \text { Financial efficiency }\end{array}$ & 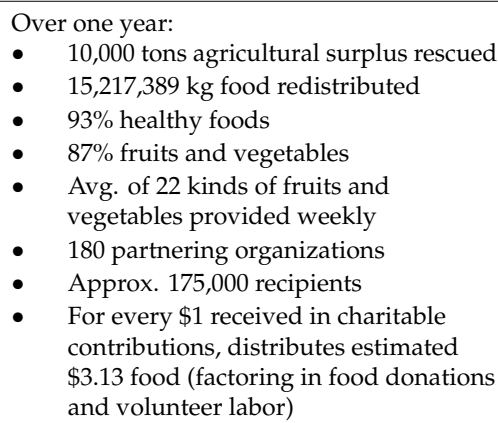 \\
\hline
\end{tabular}


Table 3. Cont

\begin{tabular}{|c|c|c|c|c|c|}
\hline Reference & Study Period & Methods & Sample & Key Metrics* & Impact \\
\hline Reynolds et al., 2015 & One year & $\begin{array}{l}\text { Input-output framework; } \\
\text { measurement }\end{array}$ & $\begin{array}{l}\text { Four food rescue } \\
\text { organizations }\end{array}$ & $\begin{array}{l}\text { Over one year: } \\
\text { - Weight of food rescued } \\
\text { - } \quad \text { Coight of food waste generated } \\
\text { - } \quad \text { Environmental impact of food rescued } \\
\text { - Comparison of food rescue to other } \\
\quad \text { disposal methods (e.g., } \\
\text { composting, landfill) }\end{array}$ & $\begin{array}{l}\text { Over one year: } \\
\text { - } \quad 18,105 \text { tons food rescued } \\
\text { - } \quad \text { Food rescue operations generate } \\
\text { approx. } 6 \mathrm{~kg} \text { food waste per ton } \\
\text { food rescued } \\
\text { - } \quad \text { Cost of US } \$ 222 \text { per ton food rescued } \\
\text { - } \quad \text { Per US dollar spent on food rescue, } \\
\text { US\$5.71 (1863 calories) food rescued } \\
\text { - Per US dollar spent on food rescue, } 6.6 \\
\text { m3 of embodied water, } 40.13 \text { MJ of } \\
\text { embodied energy, and } 7.5 \text { kilograms of } \\
\text { embodied greenhouse gasses saved } \\
\text { - Food rescue is lower cost than } \\
\text { direct purchasing }\end{array}$ \\
\hline Sewald et al., 2018 & Annually from 2012-2016 & Measurement & $\begin{array}{l}\text { One food rescue } \\
\text { organization }\end{array}$ & $\begin{array}{l}\text { In one year: } \\
-\quad \text { Weight of food rescued } \\
\text { - } \quad \text { Number of donor organizations } \\
\text { - } \quad \text { Year-over of recipient organizations } \\
\quad \text { food rescued 2012-2016 in weight of }\end{array}$ & $\begin{array}{l}\text { In 2016: } \\
\text { - } \quad>1400 \text { pounds of food per day rescued } \\
\text { - } \quad 15 \text { donor organizations } \\
\text { - } \quad \text { Approx. } 40 \text { recipient sites } \\
\text { In 2016, redistributed }>100,000 \text { more } \\
\quad \text { pounds of food than in } 2015 \\
\text { (23\% increase) }\end{array}$ \\
\hline Sisson, 2016 & Five months & $\begin{array}{l}\text { Measurement; survey of } \\
\text { clients }\end{array}$ & $\begin{array}{l}\text { Two farmers markets; } 17 \\
\text { clients surveyed }\end{array}$ & $\begin{array}{l}\text { Over } 5 \text { months: } \\
\text { - } \quad \text { Weight of food rescued } \\
\text { - } \quad \text { Cross-sectional survey measures: } \\
\text { - } \quad \text { participant report of proportion of } \\
\text { - } \quad \begin{array}{l}\text { Participant and recipient } \\
\text { organization satisfaction }\end{array}\end{array}$ & $\begin{array}{l}\text { Over } 5 \text { months } \\
\text { - } \quad 17,000 \text { lbs. fresh produce rescued } \\
\text { - } \quad \text { Cross-sectional survey results: } \\
\text { - } 70 \% \text { of participants used } 100 \% \text { of } \\
\text { - } \quad 87 \% \text { of participants shared produce } \\
\text { - } \quad 100 \% \text { agres } \\
\quad \begin{array}{l}\text { average to very good quality was } \\
\text { - } \\
\quad \text { orgonizations were satisfied }\end{array} \\
\quad\end{array}$ \\
\hline
\end{tabular}


Table 3. Cont

\begin{tabular}{|c|c|c|c|c|c|}
\hline Reference & Study Period & Methods & Sample & Key Metrics* & Impact \\
\hline Vittuari et al., 2017 & $\begin{array}{l}\text { Cross-sectional survey } \\
\text { inquiring about } \\
\text { operations over two years } \\
\text { (2014 and 2015) }\end{array}$ & $\begin{array}{l}\text { Survey of food charity } \\
\text { managers }\end{array}$ & $\begin{array}{l}61 \text { food distribution } \\
\text { initiative managers }\end{array}$ & 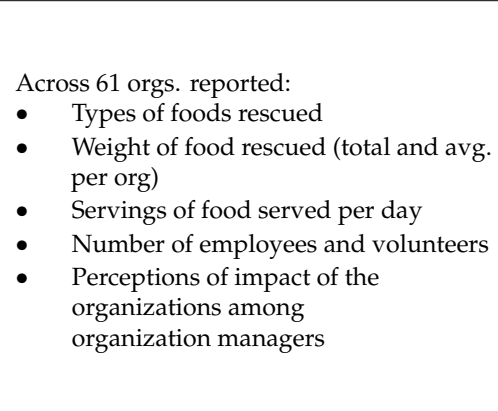 & $\begin{array}{l}\text { Across } 61 \text { orgs. reported: } \\
\text { - } \quad \text { All orgs. distributed fresh fruits and } \\
\text { vegetables, bread/bakery } \\
\text { products, non-perishables } \\
\text { - } 2014: 596 \text { tons of food rescued ( } 9.8 \text { per } \\
\text { org); } 2015: 642 \text { tons (10.5 per org) } \\
\text { - Avg. } 1095 \text { servings per org. per day } \\
\text { - } 180 \text { full-time and } 167 \\
\text { part-time/fixed-term employees; } \\
\text { 510 volunteers } \\
\text { Perceived positive social, economic and } \\
\text { nutritional impact, and } \\
\text { recipient satisfaction }\end{array}$ \\
\hline
\end{tabular}

${ }^{*}$ Relevant metrics are outcome measures included in the article that focus directly on food rescue (e.g., weight of food rescued). Articles may have also included other unrelated metrics

(e.g., other services provided by food rescue organization, barriers to food rescue), which are not included here. 
There was poor alignment between the goals of the studied interventions and the barriers/challenges highlighted in the nine contextual studies noted in Table 1. (Table 4) Two interventions addressed administrative/logistical barriers to donation, which was the most frequently mentioned concern in the identified studies. No articles focused on the second-most frequently mentioned barrier: limited funds, space, and other resources. Three focused on the challenge of improving donated food quality, which was the least frequently mentioned barrier/challenge in this literature (which does not mean it is unimportant). Additionally, the largest group of evaluations in our study (six) focused on programs addressing a challenge which was not highlighted in the contextual review: an opportunity to involve more non-traditional food sources such as farmers markets or schools.

Table 4. Studies of interventions which address top barriers to food rescue identified in prior research.

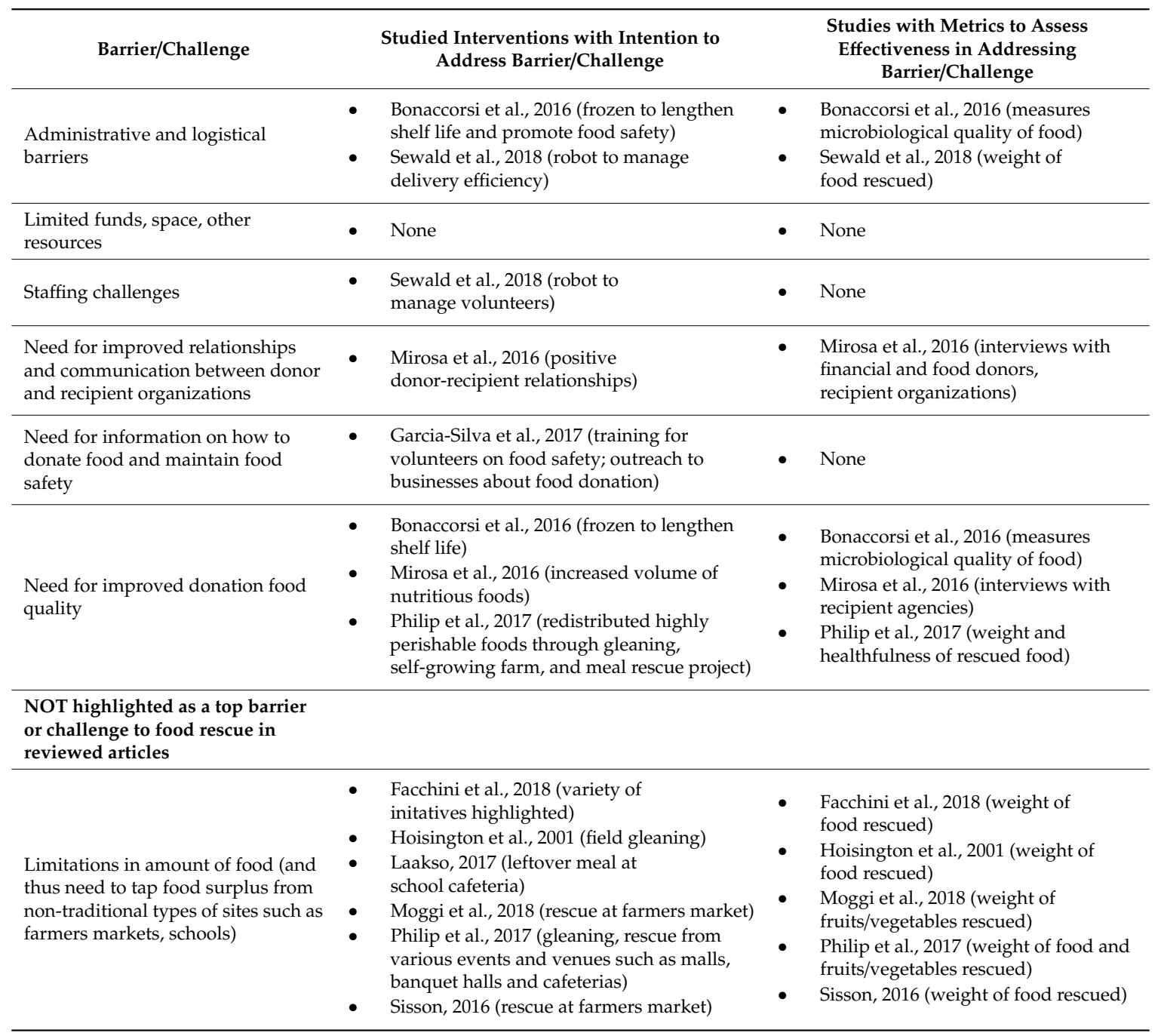

All studies used observational, rather than experimental, designs. To assess intervention impact, 14 studies used quantitative measurements (e.g., weight of food recovered, count of clients served, either collected as primary or secondary data), five used surveys, five used interviews, one used focus groups, and three used laboratory analyses of contamination. Several studies used multiple methods (e.g., interviews plus surveys). 


\subsection{Outcome Measures and Findings}

Fourteen articles reported primarily quantitative food rescue outcomes, two reported primarily qualitative outcomes, and three reported a mix of quantitative and qualitative outcomes.

\subsubsection{Outcomes: Food Recovery}

The most common metric reported, used in 14 studies, was the amount of food recovered by weight [19-32]. Comparison across studies is challenging because the time periods examined are not necessarily comparable due to seasonality and other factors. Reference time periods included: one day, two days, one month, one farm season, two school terms, 22 months, and one year. Further, some reported the amount of food recovered by a single organization or retailer, while others reported an average across several organizations or retailers.

Few articles detailed the methods used to weigh recovered food. Two articles that were focused on food recovery in supermarkets used scanned product barcodes to calculate weight of food recovered [20,21]. Beyond these, most authors used vague language to describe their methods such as "food items were weighed" but did not specify when food items were weighed (e.g., before or after cooking) and whether food items were weighed with or without packaging or including non-edible components (e.g., orange with or without peel). Only one article specified that the net weight of packaging for all packed items was subtracted in calculations [20].

One article specified the type of equipment used to weigh food (a commercial Wedderburn scale) [22]. None explained whether or how data collectors were trained or data collection methods standardized. Several authors relied on participants surveys and interviews to provide estimates of food recovered, rather than measuring quantities directly $[23,27,32]$. One article used a 41-item validated instrument, the Organizations Involved in Food Rescue Nutrition Survey, to collect this survey data [27]. Another article relied on government documents and industry reports to determine the amount of food recovered [29]. In a few cases, authors omitted any description of methods used to gather data on weight of donated food.

One article went further than total weight, reporting the amount of food at each stage of the recovery process (donated, accepted, delivered, served, consumed) [19]. The authors found that $57.6 \%$ of food donated by one U.K. retailer to two centers that served homeless clients was ultimately consumed, with the remainder being rejected or wasted for reasons including food deterioration. One article also reported the weight of food gleaned from the fields, and the proportion donated to emergency food programs (77\%) versus the proportion taken home by gleaning volunteers (23\%) [25]. Additionally, one article quantified the number of food deliveries made by a supermarket to distributors annually and monthly [21].

Four articles that reported the amount of food recovered in total weight went a step further to provide additional context by reporting the amount of food recovered in meal equivalents [21,22,24,32]. One calculated meal equivalents based on the total weight of cooked items divided by an estimated child serving size of 270 grams (270 grams was used as a proxy serving size for a child "meal", regardless of the type of food item) [22]. Another used calories per weight of each recovered item to calculate the number of portions each product could produce [21] and a third article converted the total raw weight of recovered food to meal equivalents with the standard estimate that 1.2 pounds of food equates to one meal [24]. Another article reported the number of families fed daily by rescued food but did not elaborate on whether or how family size or serving size were estimated [32].

Three articles reported the types of food recovered and two broke down the proportion by weight of food in each food category (e.g., bread, fruits and vegetables) [21,28,32]. Specifically, an Italian supermarket reported that $70 \%$ by weight of food donated was bread [21], while food distributed by an Israeli food bank was reported as $87 \%$ fruits and vegetables and $93 \%$ "healthy foods" [28]. 


\subsubsection{Outcomes: Number of Clients and Collaborators Engaged}

A small number of articles reported the number of volunteers and employees engaged [27,32] and the total [22] and average [27] number of volunteer hours logged. Two articles reported the number of clients served through food rescue operations and their collaborating agencies. One of these studies surveyed 100 U.S. organizations of varying sizes engaged in food rescue, including food pantries, emergency food programs, shelters and clinics, churches, after-school programs, and child camps, and found that organizations served on average more than 40,000 clients per month [27]. The other study reported that one large Israeli food bank served approximately 175,000 clients over one year [28]. Neither explained how the number of clients served was counted.

Three articles reported the number and type of collaborating agencies, either donor or recipient organizations, that worked with the study organization(s) of focus [24,28,31]. One reported that a U.S. county-wide coalition had recruited 50 donors and 25 food pantries over 22 months [24]. Another found that 180 organizations had partnered with an Israeli food bank [28]. The third reported that their regional food rescue network in Boulder, Colorado worked with 15 food source donor and 40 distribution sites in one year [31].

\subsubsection{Outcomes: Client and Collaborator Perceived Impacts}

Four articles reported perceptions among program recipients regarding the program's benefits, gathered through focus groups or surveys $[22,25,30,33]$. In an Australian school breakfast program using donated food, students, teachers, and parents in focus groups reported positive perceptions of program impact on student behavior, learning, and food security [22]. Surveyed participants in a field gleaning program in the US cited benefits to their diet, food budget, and knowledge related to gardening, preservation and nutrition, as well as helping others [25]. In an evaluation of a "leftover lunch" dining program in Finland for low-income adults, diners reported that the program provided both an affordable and nutritious meal and an important social opportunity [33]. Participants in a US farmer's market gleaning project reported through surveys high program satisfaction and positive impressions of produce quality [30].

Two articles reported perceptions among other stakeholders of food rescue program benefits [30,32]. In a study by Vittuari et al. surveying managers of 61 Italian food rescue initiatives, managers described positive perceptions of social, economic, and nutritional program impact, as well as perceptions of high recipient satisfaction [32]. Mirosa et al. interviewed food donors, financial donors, distribution agencies, and volunteers collaborating with one large New Zealand food rescue organization and also found perceptions among stakeholders of positive impact of participation on them or their operations [34].

\subsubsection{Outcomes: Environment and Health Impacts}

Complementary impact metrics, such as reductions in greenhouse gas emissions and water usage, were reported in three articles [21,26,29]. Two provided a detailed explanation of how environmental impacts, as well as food security impacts and monetary value of recovered food were calculated, and both found large reductions in environmental impact associated with food rescue [21,29]. The third reported an annual savings of 3,000 tons of $\mathrm{CO} 2$ associated with a US farmers market food rescue program, but did not provide any detail on how carbon emissions reductions were calculated [26].

Three articles reported the microbiological quality, hygiene, and safety of the food recovered and stored [35-37]. One, which focused on the impact of freezing recovered produce to extend its shelf-life, detected no or non-hazardous levels of pathogens in food samples, while the other two reported presence of pathogens including Listeria monocytogenes and Salmonella spp in a small subset of samples [35]. Notably, each of these articles used testing protocols and quality standards established in the study countries (Belgium and Italy), which may be different elsewhere. De Boeck et al. called for harmonization and professionalism of the food rescue chain in Belgium to better ensure 
food safety [36]. Millicevic et al. also highlighted the importance of volunteer education related to hygienic food handling practices [37].

\subsubsection{Outcomes: Financial Impacts}

Financial metrics, including program operation costs and cost savings relative to food purchase or other disposal methods, were reported by seven articles [20-22,26-29]. Two reported the financial value of food recovered, scanning the store barcode of each item recovered, and calculating its retail cost (multiplying the unit cost and the quantity). [20,21]. Another estimated the mean quantity of donations obtained per person and per household monthly and the average cost of these foods [27]. One measured the cost to operate a food rescue program, including cost of consumables, lost salary for volunteers, and transportation, calculating a total operating cost of $\$ 230,340$ [22].

Three articles calculated return on investment (ROI) of a food rescue program and found positive effects. Comparison across studies is challenging, however, as they focused on food rescue operations of different scales (a supermarket vs. a food bank vs. a food system) and used different outcome measures $[21,28,29]$. Cicatiello et al. (2016) found that one supermarket recovered food equivalent to $€ 46,000$ in one year, and the ROI was four times greater than the program operation and start-up costs [21]. Philip et al. found that for every $\$ 1$ received by a food bank in charitable contributions, the food bank distributed an estimated $\$ 3.13$ in food, factoring in non-monetized food donations and voluntary labor [28]. Reynolds et al. took a broader systems-level view, reporting that for every USD spent on food rescue in Australia, \$5.71 worth of food was rescued, and that the cost of operating a food rescue initiative was lower than direct food purchasing [29].

\subsubsection{Outcomes: Addressing Barriers and Challenges}

Table 4 summarizes the extent to which the metrics used in the studies enable even basic assessment of the programs' effectiveness in addressing identified barriers and challenges. While, as previously noted, six interventions were targeted in some way to address administrative and logistical barriers, none of the evaluations included measures to assess intervention impact on these barriers. The only areas where measures existed were to assess relationship networks, need for improved donation food quality, and tracking volume of food rescued from specific nontraditional sites.

\section{Discussion}

This systematic review provides the first synthesis of peer-reviewed studies examining food rescue interventions. Four key findings emerge.

First, included studies identified positive effects of food rescue interventions, including positive ROI, decreased environmental burden, large quantities of food rescued and clients served, and high stakeholder satisfaction. While the small body of literature is suggestive of important benefits of food rescue interventions, controlled studies and experimental evaluations are lacking. All of the included studies were observational.

Second, the fact that only nineteen articles met the broad inclusion criteria highlights the need for evaluation of a larger and broader array of traditional and complementary programs including those addressing key food rescue barriers highlighted in the literature. The reviewed studies primarily addressed donation transportation, but there was little focus on donation liability education or value-added processing and no interventions addressing policy interventions, such as standardized donation regulation or donation tax incentives. Additionally, all of the studied interventions occurred in high-income countries, and most examined interventions gathering food from supermarkets, despite the proliferation of interventions in other settings, such as farmers markets, restaurants, schools/universities, and farms. As noted above, even across these countries, social welfare systems, and thus systems and priorities for using rescued food, will differ considerably.

Third, the lack of comparability across studies highlights the need for consistent metrics and methodology to enable aggregated insights. Weight was the most common metric for quantifying 
food rescue, sometimes complemented by meal equivalents. The denominators used in terms of study length varied considerably, making comparison challenging. Studies generally did not specify how they measured weight, nor whether they included packaging, or how/whether they segmented edible vs. nonedible food. The metrics used also provide little data to allow for analysis of intervention effectiveness in addressing most of the top barriers to food rescue identified in the literature.

No studies directly compared impacts across food rescue approaches. Seven of the 19 articles examined financial metrics, but only three placed these in context of ROI $[21,28,29]$. These were unfortunately not directly comparable due to different outcome measures, but all did find positive ROI, including food rescue value equivalent to: quadruple the program start-up and operational costs; triple the value of program charitable donations; and 5.7 times that of spending.

Additionally, few studies characterized impacts of food rescue programs upon beneficiaries, such as effects on health or ability to meet food needs. While several articles analyzed environmental outcomes, finding substantial positives, the cost versus benefit of obtaining these positives was assessed only by Reynolds et al. [29]. Three articles assessed food safety and microbiological quality in food donation, suggesting a need for improved efforts, particularly in small programs and through volunteer training.

To complement this systematic assessment of peer-reviewed evaluations, we also conducted a brief scan of measurement approaches used in grey literature, such as food banks and rescue organizations websites and reports. With some exceptions, these websites and reports typically reported only a few outcome measures, such as total weight of recovered food, number of volunteers and partners engaged, and number of clients served, generally without detailed explanation of methodology [38-40]. A few also provided qualitative outcome measures, such as client, donor, or volunteer satisfaction. One archetypal example: in their annual report, Feeding America, a network of 200 food banks and pantries, presented: pounds rescued, meals provided, and percent of food that met the "Foods to Encourage" classification as healthy [41].

\section{Recommendations}

Our examination indicates a need for a consistent set of metrics for evaluating food rescue interventions. We recommend convening practitioners and researchers through an international body such as United Nations Food \& Agriculture Organization's SAVE FOOD program; or a domestic organization such as Feeding America, ReFED or the U.K. WRAP, to determine a standardized approach to evaluation metrics. The approach should address the following needs, identified based on our review, our expertise, and suggestions from other analyses such as Otten et al. [11]:

1. Develop and adapt a consistent protocol for reporting and evaluating outcomes from food rescue programs. Bellemare et al. demonstrate the substantial variation in results that would be identified based on different approaches to measurement decisions [42]. While differences in measurement approach cannot be fully avoided given diverse program goals, a consistent methodology provides a starting point for comparison. The International Food Loss and Waste Accounting and Reporting Standard [43] does not currently include methods for tracking food that is rescued rather than wasted; however, the protocol's framework and messaging could be adapted. The protocol emphasizes the need to clearly define the scope including timeframe, material type, destination where food is sent, and boundary (food category, lifecycle stage, geography, and organization). In characterizing material types, the protocol allows users to select what they are specifically including in the category of "food" based on their goals and requires clear and consistent description of these decisions. For example, studies may differentially define edibility and what food types are included. Given logistical and food safety challenges involved in weighing donations without packaging, and the current rarity of this practice, we recommend organizations consistently record weight with packaging, document this decision and the packaging type, and recognize the limitations of this approach. 
2. Improve denominator reporting. One important area for improving food rescue measurement is in presentation of denominators. Food rescue data is most commonly reported on a per client basis with a temporal indicator; for example, "the program recovered sufficient food to serve $\mathrm{X}$ clients in a one-year period." We argue that while this metric is relatively easy to obtain, it has significant flaws. The metric makes comparison across programs impossible, as different programs may provide different quantities of food per client visit. Additionally, food quantities may not be consistent even within programs, and while pantries aim to track household size, it is unknown how many people are actually fed from food obtained in one client's visit. A further potential limitation may arise regarding the time indicator if, for example, receipt of shelf-stable or frozen food is inconsistent across time so that food is not taken in and disbursed in the same month.

Many programs also report data on a per meal basis, using standard calculations of the weight of food in a meal. For example, the Feeding America network converts pounds of food to meal equivalents by dividing pounds by 1.2 (excluding water), citing a finding from the USDA's What We Eat in America 2011-2012 that an average meal is $1.2 \mathrm{lbs}$. of food [44]. While this metric is flawed because food and packaging weights are not consistent (e.g., produce and other foods with high water content will be heavier than other foods), it is a commonly used metric in the U.S. and for consistency sake, might be adopted as a standard until a stronger universal measure is developed.

3. Use consistent metrics of nutritional, health, environmental and financial impacts. Programs could use the Food Loss and Waste Value Calculator tool associated with the International Food Loss and Waste Protocol [45] to estimate nutritional and environmental impacts of food rescue programs based on food weight. This tool provides the option for some specificity by food type and, if available, origin. A more detailed nutritional data calculator is also available for the U.S. food supply based on the calculations performed in Spiker et al. [46].

We note that using nutritional data to assess potential health impacts requires additional measurement of broader household food consumption beyond what is received through a rescue program. There would be a need to assess how much of the received food was consumed, and by whom within a household and the extent to which healthy food received was a substitute versus a supplement to other healthy foods in the diet. Further challenges in assessing actual health impacts based on composition of donated food arise due to the nonlinear, time-lagged, and sometimes unclear relationships between dietary composition and health outcomes. Thus, while proximal indicators, such as healthfulness of foods distributed, can and should be measured, they should not be misinterpreted as data regarding health impacts. Other proximal client outcome measures that demonstrate program impact such as changes in access to food, access to healthy food, food security, and incidence of chronic diet-related disease could be collected using existing validated survey questions [47,48].

To estimate financial savings from food rescue in the supermarket context, where retailers have the advantage of having products with barcodes, evaluations could look to the protocol established by Cicatiello et al. [20,21]. The Environmental Protection Agency's Return on Investment Food Waste Management Calculator also provides a useful tool for businesses looking to estimate the cost competitiveness of alternatives to food waste disposal, including donation [49]. The calculator is unable to take into consideration specifics of the donation program, including detailed costs of operating the donation program, but may provide a valuable starting place for comparison.

Given complexities of assessing health, environmental, and economic impacts, we recommend that food rescue programs also consider sustained engagement with epidemiologists, economists, and other researchers with expertise in program evaluation.

4. Track food flow, including percent of food of donations that gets distributed to clients, and percent of food received by clients that is consumed. Only one study in this evaluation gathered 
food flow data [19]. It has been suggested that much of the food that is donated is never served or distributed to clients for reasons including donation of food close to spoilage or expiration. In order to track loss within the food recovery organization and identify potential intervention points, organizations should track amount, sources and types of food discarded between donation and distribution, and reasons for discard. Detailed data about consumer consumption of received food may be challenging to collect on a large scale but could be assessed in smaller scale studies. Researchers can could use one of the household waste measurement methods assessed by Zanolli et al. that best fits their program objectives [50]. Detailed data on food discards may point the way to program shifts that reduce the cost of accepting food that is not wanted or of insufficient quality.

5. Track other outcomes. A food rescue measurement protocol should also include optional modules covering factors including client satisfaction, staff perceptions, and other indicators related to implementation fidelity, program effectiveness, and sustainability. Given that some programs are intended to address particular limitations in existing food rescue programming, such as inefficiencies in logistics, measures should assess effectiveness of addressing these limitations. This additional set of indicators may be at least partly developed and modified from existing surveys and other tools.

6. Continue to perform more in-depth qualitative and quantitative assessments. Consistent indicators will go a long way toward improving the insights that can be derived from evaluations and enabling synthesis of broader messages. Data to support evidence-based decision-making about resource allocation and policy development are critically needed. To complement such research, there is also a need for further in-depth research approaches including qualitative research and data collection intended to answer questions going beyond those addressed in standard indicators.

Additional dialogue among key international stakeholders is warranted to develop globally standardized metrics that capture the impact of food rescue. These metrics should be practical and manageable to collect, incorporate some flexibility to adapt to diverse contexts, and be meaningful to those on the ground, policymakers, researchers, and funders. Given the different structure, prevalence, and role of food rescue programs across countries, the key research questions may differ, but consistent measurement will still be critical.

The strengths of this review include its novel contribution to address an important gap in the literature and the rigorous data collection method using the PRISMA framework. In terms of limitations, it is possible that additional search terms or approaches might have identified further studies that could meet inclusion criteria. The studies that summarize barriers and challenges were not systematically selected, but rather identified through the larger search strategy. In some cases, subjective decisions were required regarding whether barriers noted in the papers were distinct or duplicative, which could affect the frequency ranking of categories of barriers.

\section{Conclusions}

Food rescue programming continues to expand, including growth in traditional and complementary programs. There is a critical need for similar growth in root cause solutions that fundamentally address food insecurity and food surplus. Nonetheless, food rescue can provide many positive benefits, serving a critical need by providing food to those who need it, and providing an outlet for surplus food that might otherwise be wasted. The 19 studies we identified, all observational, suggest considerable positive effects of food rescue interventions in a range of domains, including stakeholder satisfaction, ROI, and environmental burden. At the same time, several relevant outcomes, including client health and food security measures, were omitted from existing studies, and merit further investigation.

As the sector develops, there is a great need for increased insight into the effectiveness of existing models, how to replicate and tailor interventions to specific contexts, and how to quantify impact in a way that allows comparison and helps secure funding. Additionally, there is a need to highlight 
tradeoffs faced by food rescue programs between providing increased volume of food, better quality product, and controlling costs-and to highlight solutions that have moved the needle forward in optimizing these factors. Further evaluation and dissemination may reduce the extent of duplicated effort by newly developed programs unaware of existing efforts or their experiences. This review documents a considerable need for more evaluation, evaluations of more diverse programs, and controlled and experimental studies of interventions in this sector. Further, development of consistent data collection and reporting protocols, and reporting other outcomes such as health, environmental, and financial impacts, are warranted.

Author Contributions: A.A.H. was involved in conceptualizing this study and led the work to conduct the literature search, analyze findings, and draft and edit the manuscript. R.A.N. was involved in conceptualizing this study, analyzing findings, drafting and editing the manuscript, and funding acquisition.

Funding: This research was supported by the Columbus Foundation and the Foundation for Food and Agriculture Research. A.A.H. was also supported by a Center for a Livable Future-Lerner Fellowship. The funders had no role in the design of the study; in the collection, analyses, or interpretation of data; in the writing of the manuscript, or in the decision to publish the results.

Acknowledgments: The authors thank Meg Kimmel from the Maryland Food Bank, Beth Feingold and Christine Bozlak from the University at Albany-SUNY, and Martin Bloem and Shawn McKenzie from the Johns Hopkins Center for a Livable Future for reviewing and providing feedback on the manuscript. Katy Franklin and Caroline Powell of ReFED also provided key guidance. The authors also acknowledge Catherine Turvey, Erin Biehl and Erica Johnston from the Johns Hopkins Center for a Livable Future for their contributions.

Conflicts of Interest: The authors declare no conflict of interest.

\section{References}

1. Berkenkamp, J.; Hoover, D.; Mugica, Y. Food Matters: What We Waste and How We Can Expand the Amount of Food We Rescue; National Resources Defense Council: New York, NY, USA, 2017.

2. US EPA (US Environmental Protection Agency). Food Recovery Hierarchy. Available online: https: //www.epa.gov/sustainable-management-food/food-recovery-hierarchy (accessed on 18 October 2019).

3. Gustavsson, J.; Cederberg, C.; Sonesson, U. Global Food Losses and Food Waste: Extent, Causes and Prevention; Food and Agriculture Organization of the United Nations: Rome, Italy, 2011; ISBN 978-92-5-107205-9.

4. Food and Agriculture Organization Sustainable Development Goals: 2.1.2 Severity of Food Insecurity. Available online: http://www.fao.org/sustainable-development-goals/indicators/212/en/ (accessed on 4 October 2019).

5. ReFED. A Roadmap to Reduce U.S. Food Waste by 20 Percent; ReFED: Berkeley, CA, USA, 2016.

6. Walia, B.; Sanders, S. Curbing food waste: A review of recent policy and action in the USA. Renew. Agric. Food Syst. 2019, 34, 169-177. [CrossRef]

7. Flanagan, K.; Robertson, K.; Hanson, C. Reducing Food Loss and Waste: Setting a Global Action Agenda; World Resources Institute: Washington, DC, USA, 2019.

8. ReFED. The Food Waste Innovator Database. Available online: https://www.refed.com/tools/innovatordatabase (accessed on 15 August 2019).

9. Tarasuk, V.; Eakin, J.M. Food assistance through "surplus" food: Insights from an ethnographic study of food bank work. Agric. Hum. Values 2005, 22, 177-186. [CrossRef]

10. Wingrove, K.; Barbour, L.; Palermo, C. Exploring nutrition capacity in Australia's charitable food sector. Nutr. Diet. 2017, 74, 495-501. [CrossRef]

11. Otten, J.; Getts, K.; Diedrich, S.; Benson, C. Commercial and Anti-Hunger Sector Views on Local Government Strategies for Helping to Manage Food Waste. J. Agric. Food Syst. Community Dev. 2018, 8, 55-72. [CrossRef]

12. Benson, C.; Daniell, W.; Otten, J. A qualitative study of United States food waste programs and activities at the state and local level. J. Hunger Environ. Nutr. 2018, 13, 553-572. [CrossRef]

13. Bierma, T.J.; Bazan, C.N.; Jin, G. Food Donation and Food Safety: Challenges, Current Practices, and the Road Ahead. J. Environ. Health 2019, 81, 16-21.

14. Hermsdorf, D.; Rombach, M.; Bitsch, V. Food waste reduction practices in German food retail. Br. Food J. 2017, 119, 2532-2546. [CrossRef] 
15. Schneider, F. The evolution of food donation with respect to waste prevention. Waste Manag. 2013, 33, 755-763. [CrossRef]

16. Reynolds, C.; Goucher, L.; Quested, T.; Bromley, S.; Gillick, S.; Wells, V.K.; Evans, D.; Koh, L.; Carlsson Kanyama, A.; Katzeff, C.; et al. Review: Consumption-stage food waste reduction interventions-What works and how to design better interventions. Food Policy 2019, 83, 7-27. [CrossRef]

17. Sorunmu, Y. Life Cycle Assessment of Food Loss and Waste in the Food Supply Chain: A Systematic Quantitative and Qualitative Literature, In progress.

18. Liberati, A.; Altman, D.G.; Tetzlaff, J.; Mulrow, C.; Gøtzsche, P.C.; Ioannidis, J.P.A.; Clarke, M.; Devereaux, P.J.; Kleijnen, J.; Moher, D. The PRISMA statement for reporting systematic reviews and meta-analyses of studies that evaluate health care interventions: Explanation and elaboration. PLoS Med. 2009, 6, e1000100. [CrossRef]

19. Alexander, C.; Smaje, C. Surplus retail food redistribution: An analysis of a third sector model. Resour. Conserv. Recycl. 2008, 52, 1290-1298. [CrossRef]

20. Cicatiello, C.; Franco, S.; Pancino, B.; Blasi, E.; Falasconi, L. The dark side of retail food waste: Evidences from in-store data. Resour. Conserv. Recycl. 2017, 125, 273-281. [CrossRef]

21. Cicatiello, C.; Franco, S.; Pancino, B.; Blasi, E. The value of food waste: An exploratory study on retailing. J. Retail. Consum. Serv. 2016, 30, 96-104. [CrossRef]

22. Deavin, N.; McMahon, A.T.; Walton, K.; Charlton, K. 'Breaking Barriers, Breaking Bread': Pilot study to evaluate acceptability of a school breakfast program utilising donated food. Nutr. Diet. 2018, 75, 500-508. [CrossRef]

23. Facchini, E.; Iacovidou, E.; Gronow, J.; Voulvoulis, N. Food flows in the United Kingdom: The potential of surplus food redistribution to reduce waste. J. Air Waste Manag. Assoc. 2018, 68, 887-899. [CrossRef]

24. Garcia-Silva, B.; Handler, E.; Wolfe, J. A public-private partnership to mitigate food insecurity and food waste in Orange County, California. Am. J. Public Health 2017, 107, 105. [CrossRef]

25. Hoisington, A.; Butkus, S.N.; Garrett, S.; Beerman, K. Field gleaning as a tool for addressing food security at the local level: Case study. J. Nutr. Educ. 2001, 33, 43-48. [CrossRef]

26. Moggi, S.; Bonomi, S.; Ricciardi, F. Against food waste: CSR for the social and environmental impact through a network-based organizational model. Sustainability 2018, 10, 3515. [CrossRef]

27. Mousa, T.Y.; Freeland-Graves, J.H. Organizations of food redistribution and rescue. Public Health 2017, 152, 117-122. [CrossRef]

28. Philip, D.; Hod-Ovadia, S.; Troen, A.M. A technical and policy case study of large-scale rescue and redistribution of perishable foods by the "Leket Israel" Food Bank. Food Nutr. Bull. 2017, 38, 226-239. [CrossRef]

29. Reynolds, C.J.; Piantadosi, J.; Boland, J. Rescuing food from the organics waste stream to feed the food insecure: An economic and environmental assessment of Australian food rescue operations using environmentally extended waste input-output analysis. Sustainability 2015, 7, 4707-4726. [CrossRef]

30. Sisson, L.G. Food recovery program at farmers' markets increases access to fresh fruits and vegetables for food insecure individuals. J. Hunger Environ. Nutr. 2016, 11, 337-339. [CrossRef]

31. Sewald, C.A.; Kuo, E.S.; Dansky, H. Boulder Food Rescue: An innovative approach to reducing food waste and increasing food security. Am. J. Prev. Med. 2018, 54, S130-S132. [CrossRef]

32. Vittuari, M.; De Menna, F.; Gaiani, S.; Falasconi, L.; Politano, A.; Dietershagen, J.; Segrè, A. The second life of food: An assessment of the social impact of food redistribution activities in Emilia Romagna, Italy. Sustainability 2017, 9, 1817. [CrossRef]

33. Laakso, S. Creating New Food Practices: A Case Study on Leftover Lunch Service. Food Cult. Soc. 2017, 20, 631-650. [CrossRef]

34. Mirosa, M.; Mainvil, L.; Horne, H.; Mangan-Walker, E. The social value of rescuing food, nourishing communities. Br. Food J. 2016, 118, 3044-3058. [CrossRef]

35. Bonaccorsi, G.; Lorini, C.; Pieralli, F.; Pieri, L.; Sala, A.; Tanini, T.; Nasali, M.; Dall'Olio, B.; Santomauro, F. The right to food, food donation and microbiological problems of food safety: An experience in the territory of Florence. Ann. Ist. Super. Sanita 2016, 52, 119-122.

36. De Boeck, E.; Jacxsens, L.; Goubert, H.; Uyttendaele, M. Ensuring food safety in food donations: Case study of the Belgian donation/acceptation chain. Food Res. Int. 2017, 100, 137-149. [CrossRef]

37. Milicevic, V.; Colavita, G.; Castrica, M.; Ratti, S.; Baldi, A.; Balzaretti, C.M. Risk assessment in the recovery of food for social solidarity purposes: Preliminary data. Ital. J. Food Saf. 2016, 5, 6187. [CrossRef] 
38. DC Central Kitchen. The Campus Kitchens Project. Available online: https://dccentralkitchen.org/campuskitchens-project/ (accessed on 24 July 2019).

39. Northouse, R.; Minor, E. Fighting Waste. Feeding People; Annual Report; Food Recovery Network: College Park, MD, USA, 2018.

40. McGrath, M. Food Cycle Social Impact Report; FoodCycle: London, UK, 2019.

41. Feeding America. Feeding America 2018 Annual Report: Solving Hunger Today Ending Hunger Tomorrow; Feeding America: Chicago, IL, USA, 2018.

42. Bellemare, M.F.; Çakir, M.; Peterson, H.H.; Novak, L.; Rudi, J. On the Measurement of Food Waste. Am. J. Agric. Econ. 2017, 99, 1148-1158. [CrossRef]

43. Food Loss and Waste Protocol. Food Loss and Waste Value Calculator. Available online: http://www. flwprotocol.org/why-measure/food-loss-and-waste-value-calculator/ (accessed on 15 August 2019).

44. Feeding America. The Impact of Dollars Donated to Feeding America. Available online: https://www. feedingamerica.org/ways-to-give/faq/about-our-claims (accessed on 15 August 2019).

45. World Resources Institute. Food Loss and Waste Accounting and Reporting Standard; World Resources Institute: Washington, DC, USA, 2016.

46. Spiker, M.L.; Hiza, H.A.B.; Siddiqi, S.M.; Neff, R.A. Wasted Food, Wasted Nutrients: Nutrient Loss from Wasted Food in the United States and Comparison to Gaps in Dietary Intake. J. Acad. Nutr. Diet. 2017, 117, 1031-1040.e22. [CrossRef]

47. Centers for Disease Control and Prevention. NHANES 2017: Medical Conditions Questionnaire. Available online: https://wwwn.cdc.gov/nchs/data/nhanes/2017-2018/questionnaires/MCQ_J.pdf (accessed on 15 August 2019).

48. United States Department of Agriculture. Guide to Measuring Household Food Security. Available online: https://fns-prod.azureedge.net/sites/default/files/FSGuide.pdf (accessed on 15 August 2019).

49. United States Environmental Protection Agency. Food Waste Management Calculator. Available online: https://furtherwithfood.org/resources/food-waste-management-calculator/ (accessed on 15 August 2019).

50. Zanolli, A.; McDermott, C.; Elliott, D.; Moreno, L.; Broderson, R.; Mudler, B. Oregon Wasted Food Study; Community Environmental Services: El Paso County, CO, USA, 2019.

(C) 2019 by the authors. Licensee MDPI, Basel, Switzerland. This article is an open access article distributed under the terms and conditions of the Creative Commons Attribution (CC BY) license (http://creativecommons.org/licenses/by/4.0/). 